\title{
Aplicação de um Modelo Reológico Não-Linear em Sistemas Poliméricos Dilatantes
}

\author{
Rômulo F. Navarro \\ Departamento de Engenharia de Materiais, CCT, UFPB
}

\begin{abstract}
Resumo: A dilatância é um comportamento reológico comum a vários sistemas poliméricos embora não ocorra em polímeros puros. Existem várias teorias aplicáveis à explicação desse fenômeno e alguns modelos matemáticos para o ajuste de dados experimentais de quatro de suas seis formas de ocorrência. Neste trabalho é dado enfoque à dilatância antecedida por um comportamento newtoniano e sucedida pelo comportamento pseudoplástico. Para ajustar dados constantes da literatura para sistemas desse tipo, é apresentado um modelo híbrido e não-linear. Para o teste do modelo foram utilizados dados existentes na literatura para solução de $\alpha, \omega$ poli(t-butilestireno) em óleo mineral e suspensão de partículas do copolímero de estireno com acrilato de metila em solução aquosa de poli(ácido acrílico). O modelo proposto apresenta boa concordância com os dados experimentais.
\end{abstract}

Palavras-chave: Modelo reológico não-linear, comportamentos não-newtonianos, dilatância, sistemas poliméricos.

\section{Application of Non-Linear Rheological Model to Shear-Tickening Polymeric Systems}

Abstract: Shear-thickening (dilatant) behavior is common in several polymeric systems, but not in pure polymers. There are many theories that can be used to describe shear-thickening and some mathematical models that fit experimental data of three of its six types: dilatant, newtonian-dilatant, newtonian-dilatantpseudoplastic, viscoplastic-dilatant, pseudoplastic-dilatant and newtonian-pseudoplastic-newtonian-dilatant sequences. This work deals with the behavior of newtonian-dilatant-pseudoplastic sequence behavior and proposes a hybrid, non-linear and viscous model to fit the experimental data from systems that present such rheological behavior. The systems under investigation were: a) solution of $\alpha, \omega$ poly(t-butylstyrene) in a mineral oil (3g/dl) (sample 1) and b) suspension of styrene - methyl acrylate copolymer (S-co-MA) particles $(15 \%$ vol.) in a poly(acrylic acid) (PAA) $(1 \% \mathrm{wt})$ and water solution (sample 2$)$. The proposed model agrees with the experimental data analysed.

Keywords: Non-linear rheological model, non-newtonian behavior, shear-thickening, polymeric systems.

\section{Introdução}

Dentre os fenômenos não-newtonianos, a dilatância, caracterizada pelo aumento contínuo da viscosidade aparente com a taxa de deformação ${ }^{[1]}$, é daqueles cuja ocorrência não é muito fácil de ser observada por um ou mais dos seguintes motivos:

a) exigência de um valor crítico para a taxa de deformação; b) suceder a um comportamento newtoniano;

c) preceder a um comportamento pseudoplástico;

d) exigência de superação de uma concentração crítica para fase dispersa em suspensões.

Além do acima exposto, a dilatância tem sua observação prejudicada em grande parte dos reômetros comerciais por ser freqüentemente confundida com oscilação mecânica ${ }^{[2,3]}$ ou com desvio experimental. Acrescente-se ainda o fato de que não há na literatu-

Autor para correspondência: Rômulo F. Navarro, Departamento de Engenharia de Materiais, CCT, UFPB, Av. Aprigio Veloso 882, C.P.: 10034, CEP: 58109-970, Campina Grande, PB. E-mail: romulo@dema.ufpb.br 
ra registro de ocorrência desse comportamento em polímeros puros (sintéticos ou naturais).

Ao mesmo tempo que é uma característica desejada para campos tão díspares como aplicações médicas e mecânicas, a dilatância é a causa de falhas drásticas em equipamentos da indústria de processamento que, projetados para o trabalho com materiais de uma certa viscosidade, têm seus limites máximos de segurança ultrapassados, devido ao aumento na viscosidade, durante o processamento de materiais dilatantes ${ }^{[4]}$.

Embora a conseqüência comum seja o aumento da viscosidade com a taxa de deformação, as causas da dilatância, ao contrário da pseudoplasticidade, são várias podendo depender ou não do tipo de sistema polimérico considerado: solução, suspensão, dispersão ou emulsão, além de não se restringir a um tamanho específico para as partículas ${ }^{[5]}$; à concentração da solução ${ }^{[6]}$ ou da suspensão $0^{[1]}$. Esses aspectos a fazem uma questão ainda não totalmente resolvida no campo da dinâmica dos fluidos ${ }^{[7]}$.

Desde a formulação da hipótese de Reynolds ${ }^{[1]}$, um longo tempo se passou até o surgimento da teoria de Hoffmann ${ }^{[8-10]}$. Essa nova teoria passou a ser o referencial a ser seguido e várias modificações foram feitas para adequá-la aos vários sistemas poliméricos dilatantes ${ }^{[11,12]}$. Mesmo assim, dada à particularidade de cada sistema, outras teorias tiveram que ser formuladas para explicar alguns comportamentos, embora atípicos, comuns às soluções poliméricas como:

a) dependência do caráter dilatante do tipo Arrhenius com a temperatura ${ }^{[13-15]}$;

b) formação e/ou desenvolvimento de estruturas supermoleculares durante o escoamento ${ }^{[8,16]}$;

c) conversão e/ou balanço entre ligações intra e intermoleculares ${ }^{[17-20]}$;

d) valor crítico para a taxa de deformação ${ }^{[21]}$; e

e) associações poliméricas formadas durante o escoamento $^{[22-29]}$.

Dispersões, emulsões e suspensões poliméricas dilatantes, por outro lado, embora tenham seus comportamentos reológicos atrelados à relação entre os movimentos browniano e convectivo e os efeitos hidrodinâmicos derivados desses movimentos, e às estruturas formadas como produto final dessa relação ${ }^{[1,30-35]}$, nem sempre têm o seu comportamento dilatante explicado pela teoria de Hoffmann. Em gran- de parte, isso se deve ao fato de não haver, para esses sistemas, uma relação muito definida entre tamanho e concentração de partículas da fase dispersa com a dilatância. Dados existentes na literatura enfocando esses fatores são bastante conflitantes ${ }^{[36-42]}$ e sugerem uma relação entre o comportamento das partículas sob cisalhamento (arranjo e ordenação) e o meio de dispersão utilizado ${ }^{[34,43-46]}$. Em algumas suspensões, as transformações que originam a dilatância demoram um certo tempo para ocorrer. Esse lapso de tempo favorece o surgimento de comportamentos transientes que, em geral, eclipsam a dilatância no estado estacionário ${ }^{[1,47-53]}$. Nesse caso, se a dilatância preceder a outro comportamento reológico, como nas seqüências que serão vistas mais adiante, apenas esse outro comportamento poderá ser identificado em reômetros menos sensíveis mesmo que de faixa ampla de cisalhamento.

Os critérios, exigências e peculiaridades da dilatância em sistemas poliméricos são, sem dúvidas, as principais causas da dificuldade de verificar experimentalmente esse fenômeno. Isso se torna ainda mais claro quando as formas como a dilatância se apresenta são consideradas. De uma maneira geral são estes os tipos mais comuns de ocorrência:

a) apenas dilatância (D) ${ }^{[28,34,37,42]}$;

b) dilatância precedida de um platô newtoniano $(\mathrm{ND})^{[7,54]}$

c) dilatância precedida de pseudoplasticidade $(\mathrm{PD})^{[42]}$;

d) dilatância precedida de um platô newtoniano e seguida de pseudoplasticidade e de um novo platô newtoniano (NDP) ${ }^{[13,19,21,29,35,55]}$;

e) dilatância precedida de um platô newtoniano seguido de um pseudoplástico e outro newtoniano $(\mathrm{NPND})^{[33]}$;

f) dilatância precedida de viscoplasticidade $(\mathrm{VD})^{[15,44]}$.

Enquanto os cinco primeiros tipos podem ser visualizados em curvas de viscosidade aparente versus taxa de deformação (Figura 1a), o último dos seis tipos só é possível se verificar em curvas de tensão de cisalhamento versus taxa de deformação como mostrado na Figura 1b, visto que a viscosidade aparente assume valores bastante elevados em baixas taxas de deformação.

Algumas das formas de ocorrência da dilatância anteriormente mencionadas ainda não são totalmen- 

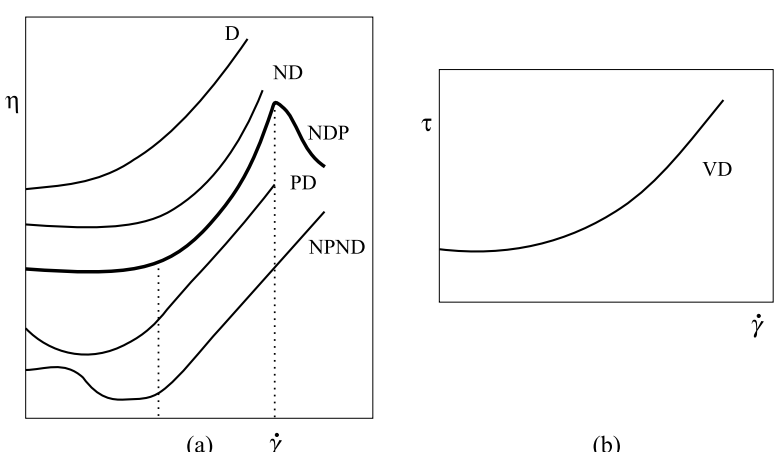

(a) $\dot{\gamma}$

(b)

Figura 1. a) Forma ilustrativa da ocorrência da dilatância em sistemas poliméricos por meio de curvas de viscosidade aparente versus taxa de deformação e b) por meio de curva de tensão versus taxa de deformação.

te aceitas. A existência de tensão residual tem sido objeto de controvérsia desde a publicação de trabalhos mostrando que esse valor desaparece em taxas de deformação próximas de zero ${ }^{[56]}$, o que pode ser o caso da viscoplasticidade apresentada pelas soluções de poliacrilamida ${ }^{[15]}$, cuja menor taxa de deformação aplicada foi da ordem de $500 \mathrm{~s}^{-1}$, ou demandam tempo para desaparecer em baixas taxas de deformação ${ }^{[57]}$, o que pode ser factível para as suspensões de biomateriais ${ }^{[4]}$ que, embora tenham sido analisadas em reômetro que pode aplicar taxa de deformação da ordem de $10^{-6} \mathrm{~s}^{-1}$, valor baixíssimo, apresentam valor muito baixo para a tensão residual entre 0,2 e $0,5 \mathrm{~Pa}$. Nos sistemas onde apenas a dilatância foi observada, a taxa mínima de deformação aplicada foi muito $\operatorname{alta}^{[37,42]}\left(200 \mathrm{~s}^{-1}\right)$; relativamente baixa ${ }^{[28]}\left(3 \mathrm{~s}^{-1}\right)$ e bai$\mathrm{x}^{[34]}\left(10^{-1} \mathrm{~s}^{-1}\right)$ o que não inviabiliza a possibilidade da existência do platô newtoniano antes do aumento da viscosidade aparente com a taxa de deformação. Por outro lado, a taxa de deformação máxima aplicada a esses sistemas $\left(71^{[28]}, 100^{[34]}, 150^{[37]} \mathrm{s}^{-1}\right)$ não inviabiliza a possibilidade de existência de um ponto de máximo e um posterior decréscimo na viscosidade aparente com a taxa de deformação. A exceção nesse caso poderia ser as suspensões de amido em água, cuja taxa de deformação máxima aplicada foi elevada, $1000 \mathrm{~s}^{-1}$. Os casos PD e NPND são poucos e merecem uma avaliação mais acurada sobre se representam um padrão ou raras exceções. Dessa forma, a seqüência NDP (em destaque na Figura 1a) destaca-se como um padrão para a representação completa da dilatância. Pois, além de estar de acordo com a maioria das teorias já apresentadas; adapta-se perfeitamente à já comprovada Teoria de Hoffmann; e é igualmente comum a sistemas não-poliméricos ${ }^{[58]}$.
Mesmo sendo objeto de controvérsias, as seqüências D, PD, NPND e VD possuem diversos modelos empíricos ou numéricos que ajustam com razoável precisão os dados experimentais. A dilatância pura e simples é característica de sistemas que obedecem à Lei da Potência de Ostwald; os formatos PD e NPND ajustam-se segundo modelo proposto por Zupancic et a ${ }^{[33]}$, o qual é uma associação do modelo de Cross com a Lei da Potência; a dilatância precedida por viscoplasticidade pode ser ajustada tanto pelo modelo de Herschel-Bulkley quanto pelo de Casson. Entretanto a sequiência NDP e sua variável ND, mesmo mais representativas para sistemas poliméricos, ainda não possuem modelos para representá-las matematicamente. O objetivo deste trabalho é desenvolver e aplicar um modelo matemático que seja aplicável à apresentação da seqüência NDP e, por conseqüência, a ND, visto que essa última está inserida na primeira.

\section{Desenvolvimento do Modelo Teórico para a Seqüência NDP}

Como pode ser visto na Figura 1a, a curva da seqüência NDP possui dois pontos críticos: o primeiro relativo ao fim do platô newtoniano e o conseqüente início da dilatância; o segundo onde a viscosidade aparente atinge seu valor máximo e a partir do qual surge o comportamento pseudoplástico. Isso sugere que a dependência global $\eta(\dot{\gamma})$ é, de fato, um somatório dos três comportamentos que ocorrem simultaneamente, cada um deles prevalecendo em um faixa de cisalhamento particular. Na Figura 2 é mostrado graficamente esse processo.

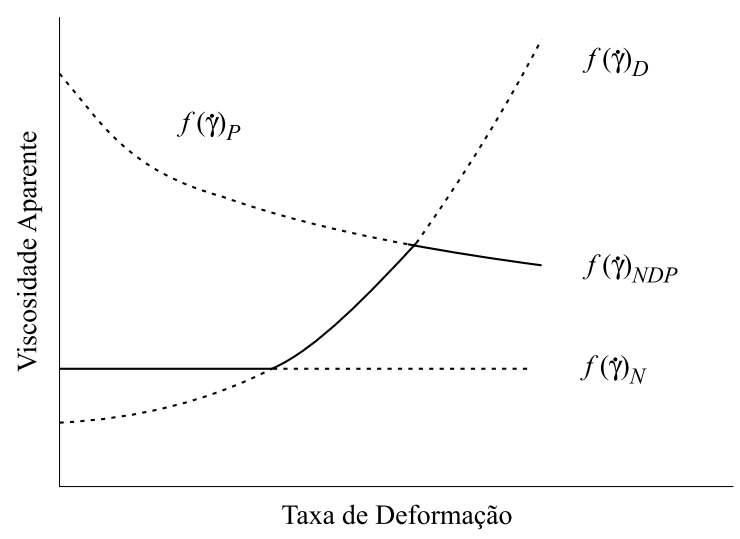

Figura 2. Curvas demonstrativas dos comportamentos newtoniano, dilatante e pseudoplástico que ocorrem simultaneamente e a curva resultante para um sistema que apresenta a seqüência NDP. 
Com base nisso, pode-se propor um modelo genérico para a dependência $\eta(\dot{\gamma})$, com seqüência NDP, da seguinte forma:

$$
\eta(\dot{\gamma})=f(\dot{\gamma})_{N D P}=f(\dot{\gamma})_{N}+f(\dot{\gamma})_{D}+f(\dot{\gamma})_{P}
$$

onde $f(\dot{\gamma})_{\mathrm{i}}$ representa cada uma das regiões da dependência global, a saber, newtoniana $(N)$, dilatante $(D)$ e pseudoplástica $(P)$. A necessidade de um valor crítico para $\dot{\gamma}$ para que $f(\dot{\gamma})_{\mathrm{D}}$ tenha início implica que $f(\dot{\gamma})_{\mathrm{N}}$ atua como retardadora de $f(\dot{\gamma})_{\mathrm{D}}$, mas não necessariamente como inibidora já que ambos os comportamentos, embora diferentes, não são antagônicos. Além disso, $f(\dot{\gamma})_{\mathrm{D}}$ leva consigo a influência de $f(\dot{\gamma})_{\mathrm{N}}$. Sendo assim, é possível a introdução de uma nova função que leve esses argumentos em consideração de forma que a equação (1) passe a ser:

$$
f(\dot{\gamma})_{N D P}=f(\dot{\gamma})_{N D}+f(\dot{\gamma})_{P}
$$

com

$$
\mathrm{F}(\dot{\gamma})_{N D}=f(\dot{\gamma})_{N}+f(\dot{\gamma})_{N} f(\dot{\gamma})_{D}
$$

Como $f(\dot{\gamma})_{\mathrm{N}}$ é constante, assumindo que

$$
f(\dot{\gamma})_{N}=\eta_{0}
$$

por conseguinte,

$$
f(\dot{\gamma})_{N D P}=\eta_{0}+\eta_{0} f(\dot{\gamma})_{D}+f(\dot{\gamma})_{p}
$$

A dependência $\eta(\dot{\gamma})$ é crescente e segue a Lei da Potência a partir de $\dot{\gamma}_{C}^{D}$ devido, segundo Hoffmann, ao congestionamento das partículas do sistema na direção do escoamento. Entretanto, o crescimento da viscosidade aparente com a taxa de deformação é inibido pela tendência dessas partículas de se orientarem na direção do escoamento sob elevadas taxas de deformação $\left(\dot{\gamma}>\dot{\gamma}_{C}^{P}\right)$ dando origem à $f(\dot{\gamma})_{P}$. De forma que uma forma adequada para $f(\dot{\gamma})_{D}$ é a seguinte:

$$
f(\dot{\gamma})_{D}=\alpha\left(\frac{\dot{\gamma}}{\dot{\gamma}_{C}^{P}}\right)^{n_{D}}
$$

garantindo que a viscosidade aparente do sistema cresça até $\dot{\gamma}=\dot{\gamma}_{C}^{P}$. Com isso, a equação (5) se transforma em:

$$
f(\dot{\gamma})_{N D P}=\eta_{0}+\eta_{0} \alpha\left(\frac{\dot{\gamma}}{\dot{\gamma}_{C}^{P}}\right)^{n_{D}}+f(\dot{\gamma})_{p}
$$

Como mencionado anteriormente, os comportamentos dilatante e pseudoplástico não são apenas opostos na forma como a viscosidade aparente varia com a taxa de deformação, mas são essencialmente conflitantes no que diz respeito ao mecanismo de ocorrência: congestionamento e alinhamento de partículas na direção do escoamento, respectivamente. Mesmo que em $\dot{\gamma}=\dot{\gamma}_{C}^{P}, f(\dot{\gamma})_{D}=f(\dot{\gamma})_{P}$, devido a essa singularidade caracterizada pela incongruência dos dois comportamentos quando ocorrem na seqüência $\mathrm{ND}$, torna-se necessário dividir a equação (7) em duas partes: uma antes e outra após $\dot{\gamma}_{C}^{P}$. Para $\dot{\gamma} \leq \dot{\gamma}_{C}^{P}$,

$$
\eta(\dot{\gamma})=\eta_{o}\left[1+\alpha \cdot\left(\frac{\dot{\gamma}}{\dot{\gamma}_{C}^{P}}\right)^{n_{D}}\right]
$$

e, como na região pseudoplástica a dependência $\eta(\dot{\gamma})$ segue a lei da potência, para $\dot{\gamma}>\dot{\gamma}_{C}^{P}$

$$
\eta(\dot{\gamma})=k_{P} \cdot \dot{\gamma}^{n_{P}-1}
$$

A divisão do modelo em duas partes não inviabiliza o modelo. Na verdade, a pseudoplasticidade surge como situação limite ao comportamento dilatante pelo fato de que o aglomerado de partículas, originado do congestionamento das mesmas na direção do escoamento, atingiu seu ponto máximo de resistência à ação do cisalhamento passando a ser desintegrado por futuros acréscimos na taxa de deformação. Comportamentos limite semelhantes têm os fluidos que apresentam viscoplasticidade, os quais são também fluidos "estruturados", e cujos modelos reológicos representativos têm partes diferentes antes e após o ponto crítico, no caso a tensão de escoamento $\left(\tau_{\gamma}\right)$.

Na equação (8), procura-se garantir a constância da viscosidade aparente inicial por uma faixa longa de taxa de deformação antes do início da fase dilatante e impor um valor máximo para a taxa de deformação em que a viscosidade aparente continue a crescer. Já a equação (9) é derivada do Modelo da Potência, cujo valor máximo inicial é igual ao valor máximo final para a viscosidade aparente na fase dilatante. Esta parte do modelo garante o decréscimo contínuo da viscosidade aparente até atingir o equilíbrio sob taxas de deformação suficientemente elevadas.

$\mathrm{O}$ significado das constantes encontra-se na Tabela 1. O modelo ora proposto será confrontado com dados existentes na literatura e com os produzidos pelos modelos da potência (equação 10) e de Cross modificado (Cross*) (equação 11) para o ajuste desses mesmos dados experimentais.

$$
\begin{aligned}
& \eta(\dot{\gamma})=k_{D} \dot{\gamma}^{n_{D}-1} \\
& \eta(\dot{\gamma})=\eta_{0}+\frac{\eta_{\infty}-\eta_{0}}{1+k_{D} \dot{\gamma}^{-n_{D}}}
\end{aligned}
$$


Tabela 1. Significado físico dos termos constantes das equações (4) a (11)

\begin{tabular}{cl}
\hline Termo & \multicolumn{1}{c}{ Significado Físico } \\
\hline$k_{D}$ & índice de consistência da região dilatante; \\
$k_{P}$ & índice de consistência da região pseudoplástica; \\
$n_{D}$ & índice da potência da região dilatante; \\
$n_{P}$ & índice da potência da região pseudoplástica; \\
$\alpha$ & constante característica do sistema; \\
$\gamma_{C}{ }^{D}$ & taxa de deformação de início da região dilatante; \\
$\dot{\gamma}_{C}^{P}$ & taxa de deformação de início da região \\
$\eta_{o}$ & viseudoplástica; \\
$\eta_{\infty}$ & viscosidade do platô newtoniade máxima. \\
\hline
\end{tabular}

\section{Resultados e Discussão}

Como pode ser visto na Figura 3, o modelo se ajusta com boa precisão aos dados experimentais, podendo ser notada uma ligeira discrepância, mais
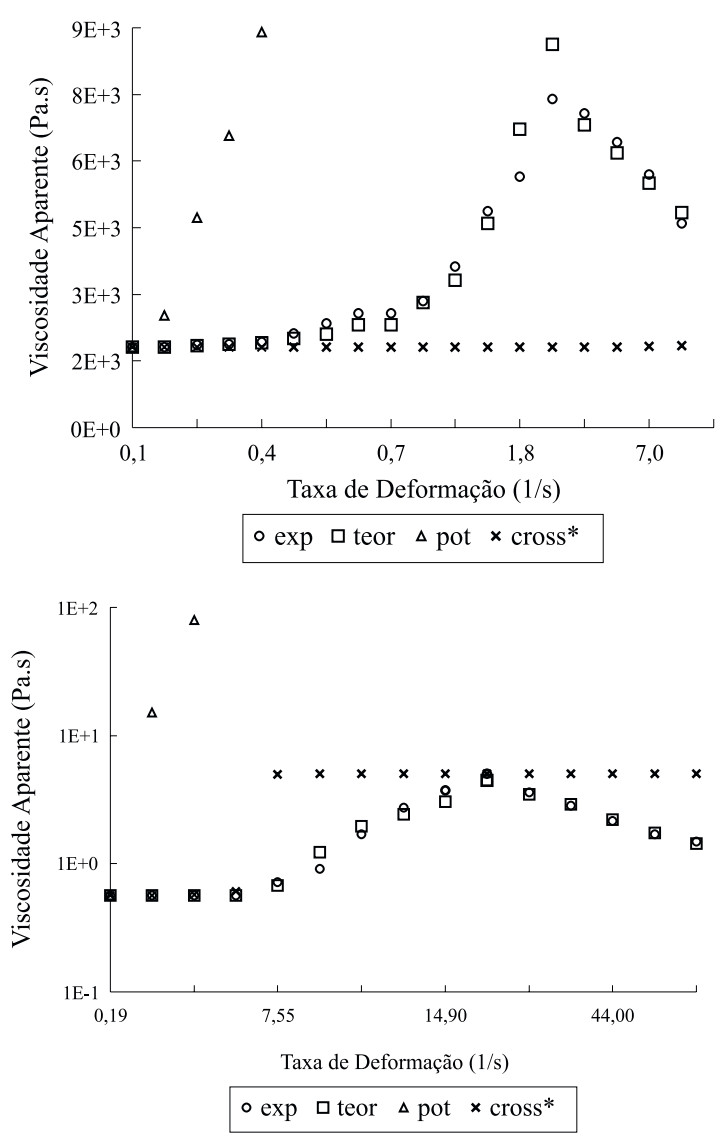

Figura 3. Aplicação do modelo proposto aos dados experimentais para (a) solução de $\alpha, \omega$ poli(t-butilestireno) em óleo mineral (3g/dl) ${ }^{[28]}$ e (b) aos dados de suspensões de copolímero de estireno-acrilato de metila (15\% em volume) em soluções aquosas de poli(ácido acrílico) $(1 \% \text { em peso })^{[35]}$
Tabela 2. Parâmetros do modelo ajustados aos dados para as amostras 1 e 2

\begin{tabular}{ccc}
\hline Parâmetro do Modelo & Amostra 1 & Amostra 2 \\
\hline$\alpha$ & 7,9765 & 6,8202 \\
$n_{D}$ & 2,3845 & 4,5952 \\
$n_{P}$ & 0,5069 & 0,2305 \\
$k_{P}\left(\right.$ Pa.s $s^{n}$ & 14.406 & 40,519 \\
$\eta_{o}($ Pa.s $)$ & $1.811,33$ & 0,567 \\
\hline
\end{tabular}

para a solução do poli(t-butilestireno) em óleo mineral (Amostra 1) do que para a suspensão do copolímero SAM em solução aquosa de PAA (Amostra 2), no valor da taxa de deformação para o início do comportamento dilatante. Entretanto, esta superestimação não causa prejuízos, tendo em vista que, prevendo um valor superior, evita futuros problemas de processamento causados por aumentos não conhecidos na viscosidade aparente.

Ambas amostras apresentam elevado grau de dilatância (ver Tabela 2) o que, pelo modelo da potência (equação 10), proporcionaria um crescimento bastante acentuado na viscosidade aparente na faixa de cisalhamento considerada, como pode ser visto nas Figuras $3 \mathrm{a}$ e $3 \mathrm{~b}$. O modelo de Cross modificado (Cross*) (equação 11), por outro lado, embora produza resultados praticamente iguais aos experimentais sob taxas de deformação mais baixas, apresenta-se inadequado a partir da taxa de deformação onde a viscosidade aparente inicia o seu crescimento mais acentuado. Dependendo do valor de $n_{D}$, o salto verificado na curva da dependência $\eta(\dot{\gamma})$ pode ocorrer sob taxas de deformação mais baixas ou mais elevadas do que as verificadas experimentalmente, quando esta dependência é ajustada pelo modelo de Cross modificado. Após esse salto, de acordo com esse modelo, o valor da viscosidade aparente do sistema deve permanecer constante por uma larga faixa para a taxa de deformação. Na prática, entretanto, nem o que seria previsto pelo modelo da potência nem pelo de Cross modificado ocorreram. Desta forma, esses modelos não podem ser utilizados em situações em que a dilatância aparece entre regiões de comportamentos newtoniano e pseudoplástico: o primeiro retardando seu início e o segundo impondo-lhe limites inferiores ao que seria usual. Por outro lado, o modelo ora proposto, por prever as duas situações acima mencionadas, ajusta-se com razoável precisão aos dados experimentais, acompanhando a dependência $\eta(\dot{\gamma})$ 
ao longo de toda a faixa de taxa de deformação utilizada para cada um dos dois sistemas considerados. Por depender da relação entre os índices da potência das regiões dilatante e pseudoplástica, o modelo proposto neste trabalho apresenta uma ligeira imprecisão na região de transição. Essa imprecisão será tanto menor quanto maiores forem os desvios do comportamento newtoniano nas duas regiões, ou seja, quando $n_{D}>>1$ quando $\gamma_{C}^{D}<\gamma<\gamma_{C}^{P}$, e $n_{P}<<1$ quando $\gamma \geq \gamma_{C}^{P}$. Esse é o caso da Amostra 2 conforme pode ser visto na Figura 3 a e Tabela 2. Apesar disso, o presente modelo apresenta-se bastante adequado à previsão do comportamento reológico sob cisalhamento de fluidos que apresentam a seqüência NDP.

\section{Conclusão}

A dilatância é um fenômeno não-newtoniano que ocorre de várias formas em sistemas poliméricos. Para várias dessas formas, existem na literatura modelos que prevêem e ajustam os dados produzidos experimentalmente. No presente trabalho foi proposto um modelo híbrido, viscoso e não-linear para adequar-se aos dados produzidos por sistemas poliméricos dilatantes que apresentam a seqüência NDP. Confrontado com dados experimentais existentes na literatura para Amostra 1 e para Amostra 2 e com os produzidos pelo modelos da potência e de Cross modificado em ajuste a esses dados experimentais, o modelo se mostrou bastante preciso e confiável.

\section{Referências Bibliográficas}

1. Navarro, R. F. - "Fundamentos de Reologia de Polímeros", EDUCS, Caxias do Sul (1987).

2. Cates, M.; McLeish, T. \& Marrucci, G.- Europhys. Lett., 21, p.451 (1993).

3. Porte, G.; Berret, J. \& Harden, J. - J. Phys II, 7, p.459 (1997).

4. Strivens, T. - “Ulmann's Encyclopedia of Industrial Chemistry", vol. B6, VCH, Berlin (1994).

5. Eirich, F. - "Thixotropy and Dilatance", ACS, Washington (1967).

6. Hu, Y.; Boltenhagen, P. \& Matthys, E. - J. Rheol., 42, p.1209 (1998).

7. Kosvintsev, S.; Riande, E.; Velarde, M. \& Guzmán, J. Polymer, 42, p.7395 (2001).
8. Hoffmann, R. - Trans. Soc. Rheol., 16, p. 155 (1972).

9. Bender, J. \& Wagner, N. - J. Rheol., 40, p. 899 (1996).

10. Bergström, L. - Colloid and Surface A: Physicochemical and Engineering Aspects, 133, p.151 (1998).

11. Bossis, S. \& Brady, R. - J. Fluid Mech., 155, p. 105 (1985).

12. Cyr, M.; Legrand, C. \& Mouret, M. - Cement and Concrete Research, 30, p. 1477 (2000).

13. Hartmann, V. \& Cressley, R. - Colloid and Surface A: Physicochemical and Engineering Aspects, 121, p.151 (1997).

14. Gamez-Corrales, R.; Berret, J.; Walker, L. \& Oberdisse, J. - Langmuir, 15, p.6755 (1999).

15. Yang, M-H. - Polymer Testing, 20, p. 635 (2001).

16. Rehage, H.; Wunderlich, I. \& Hoffmann, R. - Prog. Colloid. Polym. Sci., 72, p.51 (1986).

17. Osaki, K.; Inoue, T. \& Ahn, K. - J. of Non-Newt. Fluid Mech., 54, p.109 (1994).

18. Dupuis, D.; Lewandowski, F.; Steiert, P. \& Wolff, C. J. of Non-Newt. Fluid Mech., 54, p.11 (1994).

19. Choplin, L.; Hubert, P.; Page, A.; Houzelle, M.; Sinquin, A.; Marchal, P. \& Dellacherie, E. - Revue de L'Insitut Français du Petrole, 55, 2, p.272 (1997).

20. Tan, H. Tam, K. \& Jenkins, R. - J. of Appl. Polym. Sci., 79, 8, p.1486 (2001).

21. Bhargava, S. \& Cooper, S. - Macromolecules, 31, 2, p. 508 (1998).

22. Savins, J. - Rheol. Acta, 7, p.87 (1968).

23. Layec-Raphalen, M. \& Wolff, C. - J. Non-Newt. Fluid Mech., 1, p.159 (1976).

24. Perg, S. \& Landel, R. - J. Non-Newt. Fluid Mech., 12, p.95 (1983).

25. Maerker, J. \& Sinton, S. - J. Rheol., 30, p.77 (1986).

26. Ait-kadi, A.; Carreau, P.;\& Chauvetau, G. - J. Rheol., 31, p. 537 (1987).

27. Matthys, E. \& Saberky, R. - J. Non-Newt. Fluid Mech., 25, p.877 (1987).

28. Maus, C.; Fayt, R.; Jérôme, R. \& Teyssié, Ph. - Polymer, 36, p. 2083 (1995).

29. Navarro, R. \& França, K. - "Non-Newtonian Properties of Collagens", in: Abstracts of V International Macromolecular Colloquium, p. 132, Gramado - RS, set (1992).

Polímeros: Ciência e Tecnologia, vol. 12, no 1, p. 41-47, 2002 
30. Barnes, H. - J. Rheol., 33, p. 329 (1989).

31. D'Haene, P. - J. Colloid. Interf. Sci., 156, p. 350 (1993).

32. Bender, J. \& Wagner, N. - J. Colloid. Interf. Sci., 182, p. 171 (1995).

33. Zupancic, A.; Lapasin, R. \& Zumer, M. - Prog. Org. Coatings, 30, p. 67 (1997).

34. Mewis, J. \& Vermant, J. - Prog. Org. Coatings, 40, p.111 (2000).

35. Otsubo, Y. - Chem. Eng. Sci., 56, p. 2939 (2001).

36. Bagley, E. \& Christian, D. - J. Textures Studies, 13, p. 115 (1982).

37. Dail, R. \& Steffe, J. - J. Food Sci, 55, p.1660 (1990).

38. 3/4 - J. Food Sci, 55, p.1764 (1990).

39. Otsubo, Y. - Langmuir, 8, p.2336 (1992).

40. Laun, H. - J. Non-Newt. Fluid Mech., 54, p. 87 (1994).

41. Dintzis, F. \& Bagley, E. - J. Appl. Polym. Sci., 56, p. 637 (1995).

42. Rao, M.; Okechukwu, P.; Da Silva, P. \& Oliveira, J. Carbohydr. Polym., 33, p. 273 (1997).

43. Bourret, E. Ratsimbazafy, V.; Maury, L. \& Brossard, C. - J. Pharmacy and Pharmacology, 46, p. 538 (1994).

44. Nzihou, A.; Attins, L.; Sharrock, P. \& Ricard, A. - Powder Tech., 99, p.60 (1998).

45. Zipfel, J.; Lindner, P.; Tsianou, M.; Alexandridis, P. \& Richtering, W. - Langmuir, 15, p. 2599 (1999).

46. Duclos, R.; Bourret, E. \& Brossard, C. - International Journal of Pharmaceutics, 182, p. 145 (1999).

47. Navarro, R. - “Propriedades Reológicas de Suspensões de Alumina em Polidimetilsiloxano", Dissertação de Mestrado, Universidade Federal da Paraíba, Brasil (1989).

48. Navarro, R. \& Alsina, O. - RBE, 8, p. 79 (1991).

49. "Influencia de la Concentracion de Soluto en los Indices de Potencia y Consistencia de Suspensiones Polimericas Seudoplasticas", in: Libro de Resumes del Simposio Iberoamericano de Polimeros, p. 493, Vigo, España, jun/jul (1992).

50. Navarro, R. - "Análise Qualitativa do Efeito das Partículas da Carga na Reologia de Suspensões", in: Anais do Congresso de Pós-Graduação em Ciência e Engenharia de Materiais, p. 62, São Carlos - SP, jun (1992).

51. Varma, A. \& Chavan, V. - Carbohydr. Polym., 27, p. 63 (1995).

52. Fong, C.; Turcatte, G. \& Kee, D. - J. Food Engineering, 27, p. 63 (1996).

53. Tattiyakol, J. \& Rao, M. - Carbohydr. Polym., 43, p. 215 (2000).

54. Walkenström, P.; Nielsen, M.; Windhab, E. \& Hermansson, A. - J. Food Engineering, 42, p. 15 (1999).

55. Tan, H.; Tam, K. \& Jenkins, R. - Langmuir, 16, p. 5600 (2000).

56. Barnes, H. \& Walters, K. - Rheol. Acta, 24, p.323 (1985).

57. Shurz, J. - J. Rheol., 36, p. 1319 (1992).

58. Pal, R. - The Chem. Eng. Journal, 63, p. 195 (1996).

Recebido: 14/08/01

Aprovado: 06/03/02 